Pacific

Journal of

Mathematics

EQUIVARIANT DEFORMATIONS OF MANIFOLDS AND REAL REPRESENTATIONS

DAVIDE L. FERrario 


\title{
EQUIVARIANT DEFORMATIONS OF MANIFOLDS AND REAL REPRESENTATIONS
}

\author{
DAVIDE L. FERRARio
}

In the paper we give a partial answer to the following question: Let $G$ be a finite group acting smoothly on a compact (smooth) manifold $M$, such that for each isotropy subgroup $H$ of $G$ the submanifold $M^{H}$ fixed by $H$ can be deformed without fixed points; is it true that then $M$ can be deformed without fixed points $G$-equivariantly? The answer is no, in general. It is yes, for any $G$-manifold, if and only if $G$ is the direct product of a 2-group and an odd-order group.

\section{Introduction.}

Let $G$ be a finite group, and $M$ a compact smooth $G$-manifold. A deformation of $M$ is a homotopy of the identity $1_{M}$, i.e., a map $h: M \times I \rightarrow M$, such that $h(-, 0)=1_{M}$. We say that the deformation $h$ is fixed point free, or without fixed points if $h(x, 1) \neq x$ for all $x \in M$. If for all $t \in I$ the self-map $h(-, t)$ of $M$ is equivariant with respect to the $G$-action, then we say that $h$ is an equivariant deformation of $M$.

Mainly, the problem is to know when $M$ admits a fixed point free deformation. If $M$ is connected and the action of $G$ is trivial, then the answer is simply given by the Euler characteristic of $M$ : There is a fixed point free deformation if and only if $\chi(M)=0$. If the action of $G$ is non-trivial, any equivariant deformation of $M$ induces deformations on the subspaces fixed by the subgroups $H$ of $G$, namely $h^{H}: M^{H} \times I \rightarrow M^{H}$, for $H \subset G$, so we have the necessary condition that $M^{H}$ must be deformable without fixed points, for all $H \subset G$. With the following theorem, we show that the converse in general is not true, unless $G$ satisfies a hypothesis on its 2-Sylow subgroup.

Theorem 1.1. Let $G$ a finite group. The following propositions are equivalent:

(i) $G$ is the direct product of a 2-group $G_{2}$ and an odd-order group $G_{2^{\prime}}$ : $G=G_{2} \times G_{2^{\prime}}$.

(ii) For any real nontrivial irreducible representation $V$ of $G$, and any selfnormalizing isotropy subgroup $H$ of $G, \operatorname{dim} V^{H} \neq 1$. 
(iii) Any compact smooth $G$-manifold $M$ admits a fixed point free equivariant deformation if and only if for all isotropy types $(H)$ the submanifold $M^{H}$ admits a fixed point free deformation.

The paper is devoted to prove Theorem 1.1. The structure of the paper is closely related to the structure of the Theorem and its proof. First, in Section 2 we start by giving some preliminaries which will be needed later. Secondly, in Section 3 we prove that (i) $\Longrightarrow$ (iii) (Proposition 3.2). The proof of (iii) $\Longrightarrow$ (ii) is given in Section 4 by applying Proposition 4.1. The proof of (ii) $\Longrightarrow$ (i) is given by Proposition 8.1, in Section 8, which requires results from Sections 5, 6 and 7: The Lemmas in Section 5 and 6 are elementary. On the other hand, some results of Section 7 deeply rely on the GAP Character Tables, which are however well-known.

I would like to thank A. Dold (for his continuous help), G. Hiss (who gave me all the results in Sections 7 and 5, and more), all the people of the GAP-support (for their invaluable help - I couldn't prove this Theorem without GAP) and many others.

\section{Some preliminaries.}

Let $G$ be a finite group, acting on a space $X$. Then for each $x \in X$, we denote with $G_{x}$ the isotropy subgroup of $G$ at $x$. If $H \subset G$ is a subgroup of $G$, then we use the following notation: $N_{G}(H)$ is the normalizer of $H$ in $G$, and $W_{G}(H)$ the Weyl group $N_{G}(H) / H ; X^{H}$ is the $H$-fixed space of $X$, i.e., $X^{H}:=\{x \in X \mid H x=x\} ; X_{s}^{H}$ is the $H$-singular locus of $X$, defined by $X_{s}^{H}:=\left\{x \in X^{H} \mid G_{x} \supsetneq H\right\} ; X_{s 0}^{H}$ is the singular set of the action of the group $W_{G}(H)$ on $X^{H}$, i.e., $X_{s 0}^{H}:=\left\{x \in X^{H} \mid\left(W_{G}(H)\right)_{x} \neq e\right\}$ where $e$ denotes the identity in $W_{G}(H)$; it is easy to see that $X_{s 0}^{H} \subset X_{s}^{H}$ for all $H \subset G$. If two subgroups $H$ and $K$ of $G$ are conjugate in $G$, then we use the notation $H \sim K$. We denote with $(H)$ the conjugacy class of $H$, and with $X_{(H)}$ the $(H)$-orbit bundle of $X$, i.e., $X_{(H)}:=\left\{x \in X \mid G_{x} \sim H\right\}$. If $H$ is an isotropy subgroup of $G$, then we say that $(H)$ is an isotropy type for $X$.

Now let $X$ be a compact ENR. Then $X=\sqcup_{i} X_{i}$ is the disjoint union of its connected components $X_{i}$, with $i=1 \ldots k$. Let

$$
\underline{\chi}(X):=\left(\chi\left(X_{1}\right), \ldots, \chi\left(X_{n}\right)\right) \in \mathbb{Z}^{k}
$$

denote the vector of all the Euler characteristics $\chi\left(X_{i}\right)$.

We follow [Wi84], in order to define an equivariant Euler characteristic $\underline{\chi}_{G}$. Let $X$ be a compact equivariant Euclidean neighbourhood retract $(G$ ENR); let $A(X)$ denote the free abelian group generated by the set of all pairs $((H), C)$, where $(H)$ is an isotropy type for $X$ and $C$ is a connected component of $X_{(H)} / G$. Then $\underline{\chi}_{G}(X)$ is the unique element in $A(X)$ such 
that its $((H), C)$-component is

$$
\chi\left(X^{H}, X^{H} \backslash p_{H}^{-1} C\right)
$$

where $p_{H}: X_{H} \rightarrow X_{(H)} / G$ is the projection map. If $G$ is trivial, then $\underline{\chi}_{G}(X)=\underline{\chi}(X)$.

We will need Theorem B of [Wi84]: A compact smooth $G$-manifold $M$ admits a fixed point free equivariant deformation if and only if $\underline{\chi}_{G}(M)=0$.

We say that a finite group is 2-split when it is the direct product of a 2 -group and a group of odd order (i.e., when $G$ verifies the hypothesis of proposition (i) in Theorem 1.1).

We say that a self-normalizing subgroup $H$ of $G$ is a cutting subgroup whenever one of the following (equivalent) conditions holds:

(a) $\exists V$ real representation of $G$ such that $\operatorname{dim} V^{H}=\operatorname{dim} V^{G}+1$.

(b) $\exists V$ real representation of $G$ such that $\operatorname{dim} V^{H}=\operatorname{dim} V^{G}+1=1$.

(c) $\exists V$ real irreducible non-trivial repr. of $G$ such that $\operatorname{dim} V^{H}=1$.

Whenever a cutting subgroup $H$ of $G$ is also an isotropy subgroup with respect to the action on $V$, we call it an isotropy cutting subgroup.

If $V$ is a real representation of $G$, by tensoring $V$ with the field of complex numbers $\mathbb{C}$, we obtain a complex representation $V^{\prime}=V \otimes \mathbb{C}$ of $G$. In this case we say that $V^{\prime}$ is realizable over $\mathbb{R}$. We also have $\operatorname{dim} V^{H}=\operatorname{dim} V^{\prime H}$ for all $H \subset G$. On the other hand, if $W$ is a complex irreducible representation of $G$ of degree $n$ and character $\chi$, then one of the following sentences is true, according to the value of the second Schur Frobenius indicator

$$
\frac{1}{|G|} \sum_{g \in G} \chi\left(g^{2}\right) \text {. }
$$

(0) By restriction of scalars we obtain a real irreducible representation of degree $2 n$ and character $\chi+\bar{\chi}$.

$(+1) W=V \otimes \mathbb{C}$ for a real irreducible representation $V$ of degree $n$ and character $\chi$.

$(-1) W$ is not realizable over $\mathbb{R}$, and by restriction of scalars we obtain a real irreducible representation of degree $2 n$ and character $2 \chi$.

Moreover, every real representation can be obtained by one of the above procedures. Therefore it is readily seen that $H$ is a cutting subgroup of $G$ if and only if there exists a complex non-trivial irreducible representation $W$ of $G$ with character $\chi$ such that both the following equations hold

$$
\begin{gathered}
\frac{1}{|H|} \sum_{h \in H} \chi(h)=\left(1_{H}, \operatorname{Res}_{H}^{G} \chi\right)_{H}=1 \\
\frac{1}{|G|} \sum_{g \in G} \chi\left(g^{2}\right)=1 .
\end{gathered}
$$


Furthermore, $H$ is an isotropy subgroup for the complex representation $W=$ $V \otimes \mathbb{C}$, if and only if it is an isotropy subgroup of $G$ for $V$.

\section{Properties of 2-split groups.}

In this section we prove that (i) $\Longrightarrow$ (ii) and that (i) $\Longrightarrow$ (iii).

Proposition 3.1. Let $G$ be a 2-split group. Then $G$ has no cutting subgroups, and in particular no cutting isotropy subgroups.

Proof. Let $H \subset G$ be a self-normalizing subgroup. Because $G$ is the direct product $G_{2} \times G_{2^{\prime}}$ of a 2-group $G_{2}$ and an odd-order group $G_{2^{\prime}}$, the subgroup $H$ itself is 2-split, being the direct product of $H_{2}:=G_{2} \cap H$ and $H_{2^{\prime}}:=$ $G_{2^{\prime}} \cap H$ (cf. [Su82], Corollary p. 141). Moreover, the normalizer $N_{G}(H)$ of $H$ is the direct product of the normalizers $N_{G_{2}} H_{2}$ and $N_{G_{2^{\prime}}} H_{2^{\prime}}$. Hence $H$ is self-normalizing if and only if $H_{2}$ and $H_{2^{\prime}}$ are self-normalizing in $G_{2}$ and $G_{2^{\prime}}$ respectively. But $G_{2}$ is a 2-group, and so $H_{2}=G_{2}$.

Assume now that $H$ is a cutting subgroup, and so that $W$ is a complex irreducible nontrivial representation with second Frobenius Schur indicator $=+1$ and $\operatorname{dim} W^{H}=1$. Let $\chi$ denote its character. Because $G=G_{2} \times G_{2^{\prime}}$, we deduce that $W=W_{2} \otimes W_{2^{\prime}}$ is the tensor product of two irreducible representations of $G_{2}$ and $G_{2^{\prime}}$, with characters $\chi_{2}$ and $\chi_{2^{\prime}}$, and that $\operatorname{dim} W^{H}=\operatorname{dim} W_{2}^{G_{2}} \cdot \operatorname{dim} W_{2^{\prime}}^{H_{2^{\prime}}} . \operatorname{By} \operatorname{dim}\left(W^{H}\right)=1$, we obtain that $\operatorname{dim} W_{2}^{G_{2}}=\operatorname{dim} W_{2^{\prime}}^{H_{2^{\prime}}}=1$; hence $W_{2}$ is the trivial representation, and so $W_{2^{\prime}}$ is nontrivial.

Now, the indicator of $W$ is 1 , and so

$$
\frac{1}{|G|} \sum_{g_{2} \in G_{2}} \sum_{g_{2^{\prime}} \in G_{2^{\prime}}} \chi_{2}\left(g_{2}^{2}\right) \cdot \chi_{2^{\prime}}\left(g_{2^{\prime}}^{2}\right)=1
$$

which implies

$$
\frac{\left|G_{2}\right|}{|G|} \sum_{g_{2^{\prime}} \in G_{2^{\prime}}} \chi_{2^{\prime}}\left(g_{2^{\prime}}^{2}\right)=1
$$

because $W_{2}$ is trivial, and so $W_{2^{\prime}}$ has indicator 1 . But $G_{2^{\prime}}$ has odd order, and so $W_{2^{\prime}}$ must be trivial (it is a result of Burnside: cf. [Se77], Exercise 13.9 , p. 109), which is a contradiction.

It is a classical result that a connected compact smooth manifold admits a deformation if and only if its Euler characteristic is zero, so the following proposition is equivalent to (i) $\Longrightarrow$ (iii).

Proposition 3.2. Let $G$ be a 2-split group and $M$ a smooth compact $G$ manifold. Then $M$ admits a fixed point free equivariant deformation if and only if for all the isotropy types $(H)$ in $G$ the Euler characteristic $\chi\left(M^{H}\right)=0$ vanishes. 
Proof. It is trivial to see that if $M$ admits a fixed point free equivariant deformation, then for all the isotropy types $(H)$ the connected components of $M^{H}$ admit a fixed a fixed point free deformation, and so $\underline{\chi}\left(M^{H}\right)=0$. So let us prove the converse, i.e., that if $(\forall(H)) \underline{\chi}\left(M^{H}\right)=0$ then $M$ has an equivariant f.p.f. deformation, or equivalently $\underline{\chi}_{G}(M)=0$, because of Theorem B of [Wi84].

First we prove the following two Lemmas:

Lemma 3.3. If $G$ is 2-split and $M$ is a compact smooth $G$-manifold, then for each isotropy subgroup $H$, the codimension of $M_{s}^{H} \backslash M_{s 0}^{H}$ in $M^{H}$ is greater than 2 .

Proof. Let use point out that, because $M^{H}$ may have components of different dimension, we mean that the codimension must be evaluated componentby-component. So, let $x \in M_{s}^{H} \backslash M_{s 0}^{H}$, and $G_{x}$ the isotropy subgroup of $G$ at $x$. Assume that the codimension of $M_{s}^{H} \backslash M_{s 0}^{H}$ in the component of $x$ is 1. By the Slice Theorem (cf. [tD87] - Thm. I.5.6 p. 40) there is a real representation $V$ of $G_{x}$ such that $G \times{ }_{G_{x}} V$ is $G$-diffeomorphic to a $G$-neighbourhood of $x$ in $M$. Moreover, for all $K \subset G$ the fixed subspaces $\left(G \times_{G_{x}} V\right)^{K} \cong V^{K}$ coincide (cf. [Br72], Thm. II.3.4 p. 82), hence the codimension of $V^{G_{x}}$ in $V^{H}$ is 1 .

Because $x$ is not in $M_{s 0}^{H}$, its isotropy $\left(W_{G}(H)\right)_{x}$ with respect to the Weyl group $W_{G}(H)$ action on $M^{H}$ is trivial, and therefore $N_{G}(H) \cap G_{x}=H$. In other words, $N_{G_{x}}(H)=H$, and so $H$ is self-normalizing in $G_{x}$.

Therefore $H$ is a cutting subgroup of $G_{x}$. But $G_{x}$ is a subgroup of $G$, which is 2-split, and so $G_{x}$ itself is 2-split; this implies that $G_{x}$ cannot have cutting subgroups, because of Proposition 3.1, a contradiction. Hence the codimension must be greater than 1 .

Lemma 3.4. If for all isotropy types $(H)$ the Euler characteristic vanishes, $\underline{\chi}\left(M^{H}\right)=0$, then for all $H, \underline{\chi}\left(M_{s}^{H}\right)=0$.

Proof. We can prove it by a kind of induction on isotropy subgroups: let us order the isotropy subgroups (they are finite) by inclusion, i.e., $H<K$ if and only if $H \subset K$. We will denote with the symbols of $\mathcal{F}$ or $\mathcal{F}_{i}$, with $i$ integer, the subsets of the (partially ordered) set of isotropy groups with the property that if $H \in \mathcal{F}$ and $K \supset H$ then $K \in \mathcal{F}$. For such an $\mathcal{F}$, let $M^{\mathcal{F}}$ denote the union $\cup_{H \in \mathcal{F}} M^{H}$, and $\# \mathcal{F}$ the number of its elements.

We make induction on $\# \mathcal{F}$ to prove that $\chi\left(M^{\mathcal{F}}\right)=0$ for all $\mathcal{F}$. If $\# \mathcal{F}=1$, then it has just one isotropy subgroup, and by assumption $\underline{\chi}\left(M^{\mathcal{F}}\right)=0$. Assume that $\chi\left(M^{\mathcal{F}}\right)=0$ for all $\mathcal{F}$ with $\# \mathcal{F} \leq k$, and let $\mathcal{F}_{1}$ be a family such that $\# \mathcal{F}_{1}=k+1$. In $\mathcal{F}_{1}$ there are a finite number of minimal elements, say $H_{0}, \ldots, H_{m}$. If $m=0$, i.e., the minimal element is unique, then $M^{\mathcal{F}_{1}}=M^{H_{0}}$ and so by hypothesis $\chi\left(M^{\mathcal{F}_{1}}\right)=0$. If $m \geq 1$ then let $\mathcal{F}_{2}:=\mathcal{F}_{1} \backslash\left\{H_{0}\right\}$. By induction hypothesis, $\chi\left(M^{\mathcal{F}_{2}}\right)=0$; moreover, $M^{\mathcal{F}_{1}}=M^{\mathcal{F}_{2}} \cup M^{H_{0}}$ and the 
intersection $M^{\mathcal{F}_{2}} \cap M^{H_{0}}=M^{\mathcal{F}_{3}}$, where $\mathcal{F}_{3}$ is the family of isotropy groups $K \in \mathcal{F}_{2}$ such that $K \supset H_{0}$. Because $H_{0}$ is not the only minimal element, $\# \mathcal{F}_{3} \leq k$, and so $\chi\left(M^{\mathcal{F}_{3}}\right)=0$. Moreover, each $M^{\mathcal{F}_{i}}$ is a union of smooth transversal submanifolds in $M$, and therefore

$$
\underline{\chi}\left(M^{\mathcal{F}_{1}}\right)=\underline{\chi}\left(M^{\mathcal{F}_{2}}\right)+\underline{\chi}\left(M^{H_{0}}\right)-\underline{\chi}\left(M^{\mathcal{F}_{3}}\right)=0
$$

which is the thesis. We can now apply this result to the Lemma, because $M_{s}^{H}=M^{\mathcal{F}}$ where $\mathcal{F}$ is the family of all the isotropy subgroups strictly greater than $H$, and this completes the proof.

Remark 3.5. With almost the same proof as Lemma 3.4, it is possible to prove that if $\chi\left(M^{H}\right)=0$ for all the isotropy subgroups $H$ in $G$, then $\underline{\chi}\left(M^{K}\right)=0$ for any subgroup $K$ of $G$.

Now we are able to prove Proposition 3.2. Recalling the definition of $\underline{\chi}_{G}(M)$, we must prove that for each isotropy subgroup $H$ of $G$ and each connected component $C$ of $M_{(H)} / G$, the relative Euler characteristic

$$
\chi\left(M^{H}, M^{H} \backslash p_{H}^{-1} C\right)
$$

is zero. First, let us notice that $M_{(H)} / G \cong M_{H} / W$, where $W:=W_{G}(H)$, and so we may take $p_{H}: M_{H} \rightarrow M_{H} / W$, and $C$ is a connected component of $M_{H} / W$. Now let us consider the projection $q_{H}: M^{H} \rightarrow M^{H} / W$. If $D$ is a connected component of $M^{H} / W$, then it contains a connected component $C$ of $M_{H} / W$, because $M_{H}$ is open and dense in $M^{H}$. Furthermore, the inclusion $M_{H} / W \rightarrow\left(M^{H} \backslash M_{s 0}^{H}\right) / W$ induces a bijection on connected components, because of Lemma 3.3 (let us note that $M_{H}=M^{H} \backslash M_{s}^{H}$ ); the same happens to the inclusion $\left(M^{H} \backslash M_{s 0}^{H}\right) / W \rightarrow M^{H} / W$ because the action of $W$ is smooth, and $M_{s 0}^{H}$ is exactly the singular locus for its action. Hence, the connected components of $M_{H} / W$ are the intersection with $M_{H} / W$ of the connected components of $M^{H} / W$.

Therefore we need to prove that $\chi\left(M^{H}, M^{H} \backslash p_{H}^{-1}\left(D \cap M_{H} / W\right)\right)=0$ for all connected components $D$ of $M^{H} / W$. Let us define $C:=D \cap M^{H} / W$. Because $p_{H}^{-1} C=q_{H}^{-1}(D) \cap M_{H}$, the complement $M^{H} \backslash p_{H}^{-1} C=\left(M^{H} \backslash q_{H}^{-1} D\right) \cup$ $M_{s}^{H}$. So, by the exact sequence of the triple $\left(M^{H}, M^{H} \backslash q_{H}^{-1} D \cup M_{s}^{H}, M^{H} \backslash\right.$ $\left.q_{H}^{-1} D\right)$, we obtain that $\chi\left(M^{H}, M^{H} \backslash q_{H}^{-1} D\right)=\chi\left(M^{H}, M^{H} \backslash p_{H}^{-1} C\right)+\chi\left(M^{H} \backslash\right.$ $\left.q_{H}^{-1} D \cup M_{s}^{H}, M^{H} \backslash q_{H}^{-1} D\right)$. By a simple excision argument, the last term is equal to $\chi\left(M_{s}^{H}\right)$, which is zero by Lemma 3.4 , and so for each connected component $C$ the equality

$$
\chi\left(M^{H}, M^{H} \backslash q_{H}^{-1} D\right)=\chi\left(M^{H}, M^{H} \backslash p_{H}^{-1} C\right)
$$

holds true. But again applying excision, we see that $\chi\left(M^{H}, M^{H} \backslash q_{H}^{-1} D\right)=$ $\chi\left(q_{H}^{-1} D\right)$; but $q_{H}^{-1} D$ is a disjoint union of connected components of $M^{H}$, and 
by the assumption $\chi\left(M^{H}\right)=0$ each of them has Euler characteristic zero. Therefore

$$
\chi\left(M^{H}, M^{H} \backslash p_{H}^{-1} C\right)=\chi\left(q_{H}^{-1} D\right)=0
$$

which completes the proof.

\section{Cutting subgroups and equivariant deformations.}

In this section we prove that (iii) $\Longrightarrow$ (ii), which follows from the following proposition.

Proposition 4.1. If $G$ has a cutting isotropy subgroup $H$, then there is a compact connected smooth $G$-manifold $M$, without boundary, such that

$$
\underline{\chi}_{G}(M) \neq 0
$$

and

$$
\underline{\chi}\left(M^{H}\right)=0
$$

for every isotropy subgroup $H$ of $G$.

Proof. Let $V_{0}$ be the real irreducible non-trivial representation of $G$, such that $x \in V_{0} \backslash 0$ is a generator of $V_{0}^{H} \cong \mathbb{R}, H$ is self-normalizing and $G_{x}=H$ is the cutting isotropy subgroup. Let $V:=V_{0}+\mathbb{R}^{3}$, i.e., $V$ is equal to $V_{0}$ plus three times the trivial representation on $\mathbb{R}$. Let $T$ denote the 2-dimensional torus and $C$ a closed simple smooth curve in $T$ bounding an (open) disc $B$. We can embed the pair $(T, B)$ in $\left(V^{H}, V^{G}\right) \cong\left(\mathbb{R}^{4}, \mathbb{R}^{3}\right)$ in a way such that $T \backslash V^{G}$ is the disjoint union of $B$ and the complement of the closure of $B$. Moreover, we can assume that any small enough smooth regular neighbourhood of $T$ in $V^{H}$ is a trivial $D^{2}$-bundle on $T$, and $T \cap V^{G} \approx C \times D^{2}$.

Now let $G T$ be the union of all the images $g T$ in $V$, where $g$ ranges in $G$. It is a $G$-space, and let $X$ be a $G$-regular neighbourhood of $G T$ in $V$, $G$-deformable to $G T$. Without loss of generality, we can assume that $X$ is a smooth compact connected $G$-manifold with boundary, of dimension $\operatorname{dim} V+3$. Let $M=2 X$ be $G$-space obtained by joining two copies of $X$ along the boundary $\partial X$, with the identity identification map on $\partial X$. Then $M$ is a compact connected smooth $G$-manifold without boundary.

For every isotropy subgroup $K$, the Euler characteristic satisfies $\underline{\chi}\left(M^{K}\right)=$ $2 \underline{\chi}\left(X^{K}\right)-\underline{\chi}\left(\partial X^{K}\right)$. Because $\underline{\chi}\left(\partial X^{K}\right)=\left(1+(-1)^{n}\right) \underline{\chi}\left(X^{K}\right)$, where $n:=$ $\operatorname{dim} X^{K}-1$ (cf. [Do72], Corollary VIII.8.8, p. 299), we have that $\underline{\chi}\left(M^{K}\right)=$ $\left(1-(-1)^{n}\right) \underline{\chi}\left(X^{K}\right)$. But $\underline{\chi}\left(X^{K}\right)=\chi\left(G T^{K}\right)$, and $\chi\left(G T^{K}\right)=0$ for all $K$. Therefore $\underline{\chi}\left(M^{K}\right)=0$ for all isotropy subgroup $K$.

On the other hand, $H$ is self-normalizing, so the Weyl group $W:=W_{G} H$ is the trivial group. Hence the projection $p_{H}: M_{H} \rightarrow M_{H} / W=M_{H}$ is just the identity map. The connected components of $M_{H}$ are now two: $C_{+} \approx B \times S^{2}$, where $S^{2}$ denotes the 2 -sphere, and $C_{-} \approx(T \backslash \bar{B}) \times S^{2}$. Let us compute 
$\chi\left(M^{H}, M^{H} \backslash p_{H}^{-1} C_{+}\right)$: it is equal to $\chi\left(T \times S^{2},(T \backslash \bar{B}) \times S^{2}\right)$ and therefore to $2 \chi(T, T \backslash \bar{B})=2 \chi(T)-2 \chi(T \backslash \bar{B})=2$. Again, $\chi\left(M^{H}, M^{H} \backslash p_{H}^{-1} C_{-}\right)=-2$. These equalities imply that $\underline{\chi}_{G}(M) \neq 0$ which is the thesis.

\section{Further preliminaries.}

Lemma 5.1. Let $G$ be a finite group, $N \triangleleft G$ a normal subgroup and $q$ : $G \rightarrow G / N$ the quotient homomorphism. If $K \subset G / N$ is a cutting (isotropy) subgroup of $G / N$, then $q^{-1} K$ is a cutting (isotropy) subgroup of $G$.

Proof. If $G / N$ acts on the vector space $V$, then this action induces an action of $G$ on $V$, and if $x \in V$, then its isotropy subgroup $G_{x}$ of $G$ is exactly the counter-image $q^{-1}(G / N)_{x}$ of the isotropy subgroup at $x$ of $G / N$. Moreover, if $K \subset G / N$ is self-normalizing, then $q^{-1} K$ is self-normalizing in $G$.

Lemma 5.2. If $N \triangleleft G$ is a normal subgroup of $G$, and $H \subset G$ is a subgroup such that

(i) $N \cap H$ is a cutting (isotropy) subgroup of $N$;

(ii) $H N=G$

then $H$ is a cutting (isotropy) subgroup of $G$.

Proof. The normalizer of $H$ in $G$ is $N_{G}(H) \subset H N_{N}(H \cap N)$ because $G=$ $H N$, and the last is $H(H \cap N)=H$, hence $H$ is self-normalizing. Let $V$ be a real nontrivial representation of $N$ such that $\operatorname{dim} V^{N \cap H}=\operatorname{dim} V^{N}+1=1$, i.e., if $\chi$ denotes its character,

$$
\left(1_{H}, \operatorname{Res}_{N \cap H}^{N} \chi\right)_{H \cap N}=1 .
$$

Then, if $W$ denotes the induced representation $W:=\operatorname{Ind}_{N}^{G} V$, with character $\operatorname{Ind}_{N}^{G} \chi$, we know that $W$ is real and non-trivial; using Frobenius reciprocity, we obtain

$$
\begin{aligned}
\operatorname{dim} W^{G} & =\left(1_{G}, \operatorname{Ind}_{N}^{G} \chi\right)_{G} \\
& =\left(1_{N}, \chi\right)_{N}=\operatorname{dim} V^{N}=0 .
\end{aligned}
$$

Furthermore,

$$
\begin{aligned}
\operatorname{dim} W^{H} & =\left(1_{H}, \operatorname{Res}_{H}^{G} \operatorname{Ind}_{N}^{G} \chi\right)_{H} \\
& =\left(\operatorname{Ind}_{H}^{G} 1_{H}, \operatorname{Ind}_{N}^{G} \chi\right)_{G}=\left(\operatorname{Res}_{N}^{G} \operatorname{Ind}_{H}^{G} 1_{H}, \chi\right)_{N} ;
\end{aligned}
$$

because $\operatorname{Res}_{N}^{G} \operatorname{Ind}_{H}^{G} 1_{H}=\operatorname{Ind}_{H \cap N}^{N} 1_{H \cap N}$ (cf. [Se77], Proposition 22, p. 58), the latter is equal to

$$
\begin{aligned}
\left(\operatorname{Ind}_{H \cap N} 1_{H \cap N}, \chi\right)_{N} & =\left(1_{H \cap N}, \operatorname{Res}_{H \cap N}^{N} \chi\right)_{H \cap N} \\
& =\operatorname{dim} V^{H \cap N}=1
\end{aligned}
$$

and so $H$ is a cutting subgroup of $G$. 
If $H \cap N$ is an isotropy subgroup, say of $x \in V^{H \cap N}$, then the element $y:=\sum_{r \in[G: N]} r x \in W$, where $r$ ranges over a system of coset representatives of $G / N=H / H \cap N$ in $G$ such that $r \in H$, has isotropy exactly $H$, and hence the thesis.

Lemma 5.3. Let $N \triangleleft G$ a normal subgroup of $G$. If the normalizer in $N$ of a p-Sylow subgroup of $N$ is a cutting subgroup, then $G$ has an isotropy cutting subgroup.

Proof. Let $P$ be a $p$-Sylow subgroup of $N$, and let us assume that $N_{N}(P)$ is a cutting subgroup of $N$. Then the normalizer $N_{G}(P)$ in $G$ of $P$ has the following properties:

- $N_{G}(P) N=G$ (cf. [Su82], Theorem 2.7, p. 99).

- $N_{G}(P) \cap N=N_{N}(P)$.

- If $H \supset N_{G}(P)$ then $H$ is self-normalizing (because $N_{G}(P) \supset N_{G}(S)$ [Su82], Theorem 2.6, p. 98 - and hence apply [Su82], Th.2.8, p. 99).

Hence we can apply Lemma 5.2 , to prove that $H:=N_{G}(P)$ is a cutting subgroup of $G$. Let $V$ denote the irreducible non-trivial real representation of $G$, such that $\operatorname{dim} V^{H}=1$. If $x$ is a generator of $V^{H}$, its isotropy subgroup $G_{x} \supset H$, and hence it is self-normalizing. This is the wanted cutting isotropy subgroup.

Lemma 5.4. Let $N \triangleleft G$ a normal subgroup of $G$. If $M$ is a maximal selfnormalizing subgroup of $G$ such that $M \cap N$ is a maximal subgroup of $N$, and $M \cap N$ is a cutting subgroup of $N$, then $M$ is a cutting isotropy subgroup of $G$.

Proof. Because $M \cap N$ is maximal, and cutting, it must be a cutting isotropy subgroup of $N$. Moreover, $M N \supsetneq M$ and hence $M N=G$ by maximality of $M$. Therefore we can apply Lemma 5.2.

\section{Solvable groups.}

Lemma 6.1. Let $G=E \rtimes \mathbb{Z}_{2}$ be the semidirect product of an elementary abelian p-group $E$ and the 2-cyclic group $\mathbb{Z}_{2}$. If $G$ is not 2 -split, then the normalizer $N_{G}\left(\mathbb{Z}_{2}\right)$ is a cutting subgroup of $G$.

Proof. Let $\theta: E \rightarrow E$ be the automorphism of $E$ given by conjugation with the generator of $\mathbb{Z}_{2}$. Because $G$ is not 2-split, the prime $p$ is odd and $\operatorname{Fix}(\theta) \neq E$. Also, $\operatorname{Fix}(\theta)$ is a normal subgroup of $G$, and $G / \operatorname{Fix}(\theta)$ is equal to $(E / \operatorname{Fix}(\theta)) \rtimes \mathbb{Z}_{2}$, where $\theta$ acts as an automorphism of $E^{\prime}:=E / \operatorname{Fix}(\theta)$ without fixed points (different from $0 \in E^{\prime}$ - we use additive notation for $E$ ). It is easy to see that by applying Lemma 5.1 to the projection $G \rightarrow E^{\prime} \rtimes \mathbb{Z}_{2}$, it suffices to prove only the case $\operatorname{Fix}(\theta)=0$. This implies $\theta(e)=-e$ for all $e \in E$. Therefore, $G$ contains a normal subgroup $D_{2 p}$ isomorphic to the 
dihedral group of order $2 p$. For $D_{2 p}$ the proposition is true, hence we can apply Lemma 5.3 to obtain that $N_{G}\left(\mathbb{Z}_{2}\right)$ is a cutting subgroup of $G$.

Lemma 6.2. Let $G=D \rtimes \mathbb{Z}_{2}$ be the semidirect product of an odd-order finite group $D$ and the 2 -cyclic group $\mathbb{Z}_{2}$. If $G$ is not 2 -split, then $N_{G}\left(\mathbb{Z}_{2}\right)$ is a cutting subgroup of $G$.

Proof. As in the proof of Lemma 6.1, let $\theta: D \rightarrow D$ the automorphism given by conjugation with the generator of $\mathbb{Z}_{2}$. Because $G$ is not 2-split, $\operatorname{Fix}(\theta) \neq D$. By the Feit-Thompson Theorem, $D$ is solvable. Let us prove the assertion by induction on the composition length $l(D)$ of $D$, i.e., the length of a composition series in $D$ (cf. [Su82], p. 43). If $l(D)=1$, then it follows from Lemma 6.1. Assume it holds whenever $G=D^{\prime} \rtimes \mathbb{Z}_{2}$ where $D^{\prime}$ is any odd order group with $l\left(D^{\prime}\right) \leq l(D)-1$. Let $D_{0}$ be a characteristic nontrivial subgroup of $D$. Then $D_{0} \rtimes \mathbb{Z}_{2}$ is a normal subgroup of $G$. Now, if $G_{0}:=D_{0} \rtimes \mathbb{Z}_{2}$ is not 2-split, then by induction hypothesis the normalizer $N_{G_{0}}\left(\mathbb{Z}_{2}\right)$ is a cutting subgroup of $G_{0}$, and so we can apply Lemma 5.3 to finish. On the other hand, if $G_{0}$ is 2-split, then $G / D_{0}=\left(D / D_{0}\right) \rtimes \mathbb{Z}_{2}$ cannot be 2-split: Otherwise for all $x \in D$ we should have $x^{-1} \theta(x) \in D_{0}$ where $D_{0} \subset \operatorname{Fix}(\theta)$, which implies $\theta(x)=x$ for all $x$ (i.e., $G 2$-split). But again we can apply the induction hypothesis, with Lemma 5.1, to see that $N_{G}\left(\mathbb{Z}_{2}\right)$ is a cutting subgroup of $G$.

Lemma 6.3. Let $G$ be a 2-nilpotent group (i.e., the 2-Sylow subgroup of $G$ has a normal complement). If $G$ is not 2-split, then it has a cutting isotropy subgroup.

Proof. By hypothesis $G=D \rtimes S_{2}$, the semidirect product of the (odd-order) normal complement $D$ and the 2-Sylow subgroup $S_{2}$. Let $\alpha: S_{2} \rightarrow \operatorname{Aut}(D)$ be the homomorphism which defines the semi-direct product. Because $G$ is not 2-split, $\operatorname{ker} \alpha \neq S_{2}$; also, the quotient $G / \operatorname{ker} \alpha$ is still 2-nilpotent and not 2 -split. By Lemma 5.1 therefore it suffices to consider the case $\operatorname{ker} \alpha=1$. Because $S_{2}$ is a 2-group, there exists a normal 2-cyclic subgroup $\mathbb{Z}_{2} \triangleleft S_{2}$. Now, $N:=D \rtimes \mathbb{Z}_{2}$ is a normal subgroup of $G$, and fulfills the assumptions of Lemma 6.2. Therefore $N_{N}\left(\mathbb{Z}_{2}\right)$ is a cutting subgroup. Hence, by Lemma $5.3, G$ has a cutting isotropy subgroup.

Lemma 6.4. Let $G=E \rtimes F$ be the semidirect product of an elementary abelian 2-group $E$ and an elementary abelian p-group $F$ for an odd prime $p$. If $G$ is not 2-split, then $N_{G}(F)$ is a cutting subgroup of $G$.

Proof. Because $G$ is not 2-split, the intersection $\cap_{f \in F} \operatorname{Fix}(f) \neq E$, where $\operatorname{Fix}(f)$ denotes the subgroup of $E$ fixed by the automorphism induced by conjugation with $f \in F$ (i.e., the centralizer of $f$ in $E$ ). Let $e \in E \backslash \cap_{f}$ $\operatorname{Fix}(f)$. Let $V:=\mathbb{R}$ be a the representation of $E$ such that $\chi(e)=-1$, and $\chi\left(\cap_{f} \operatorname{Fix}(f)\right)=1$, where $\chi$ is the character of $V$. If we consider the induced 
representation $W:=\operatorname{Ind}_{E}^{G} V$, then it is easy to see that $\operatorname{dim} W^{H}=1$, and $\operatorname{dim} W^{G}=0$, where $H:=N_{G}(F)$. Hence the thesis.

Lemma 6.5. Let $G=E \rtimes F$ be the semidirect product of an elementary abelian p-group, and a 2-split group $F$. If $G$ is not 2-split, then it has a cutting isotropy subgroup.

Proof. If $p$ is an odd prime, then $G$ is 2-nilpotent, because $F$ is 2-split, and so we can apply Lemma 6.3. If $p=2$, then $F=F_{2} \times F_{2^{\prime}}$, where $F_{2}$ is a 2group and $F_{2^{\prime}}$ an odd-order group. Because $G$ is not 2 -split, in particular it cannot be a 2-group, and hence $F_{2^{\prime}}$ is not trivial. Now, if $\alpha: F_{2^{\prime}} \rightarrow$ Aut $E$ is the homomorphism given by the semidirect product (conjugation), as before we know that ker $\alpha \neq F_{2^{\prime}}$; therefore, up to using Lemma 5.1, we can assume ker $\alpha=1$. Let $N$ be a minimal normal subgroup of $F_{2^{\prime}}$. It is also a minimal normal subgroup of $F$, and $E \rtimes N$ is a normal subgroup of $G$. But $N$ is an elementary $q$-abelian group, with $q$ odd prime, and so we can apply Lemma 6.4 to $E \rtimes F$. It is not 2 -split because we assume $\operatorname{ker} \alpha=1$. Furthermore, the conclusion follows by Lemma 5.3.

Proposition 6.6. Let $G$ be a finite solvable group. Then $G$ has a cutting isotropy subgroup if and only if $G$ is not 2-split.

Proof. We already know that if $G$ is 2 -split then it has no cutting subgroups (Proposition 3.1). So let us assume that there are not 2-split groups without cutting isotropy subgroups. Let $G$ be the minimal example, with respect to the number of elements. By assumption, $G$ is solvable, and hence it has a nontrivial minimal normal subgroup $N$, which is elementary $p$-abelian. The quotient $G / N$ is 2-split, otherwise we could use Lemma 5.1 to find a cutting isotropy subgroup in $G$, by minimality of $G$. Moreover, $G$ cannot be 2nilpotent (by Lemma 6.3), while $G / N$ is 2 -nilpotent. By an easy consequence of Proposition 9.5.2 of [Ro82], p. 270, $N$ must have a complement $F \cong$ $G / N$, hence $G$ is the semidirect product of an elementary abelian $p$-group and a 2-split complement $F$. But these are the assumptions of Lemma 6.5, and so $G$ has a cutting isotropy subgroup: a contradiction.

\section{Almost simple groups.}

7.1. Alternating groups. The simple alternating groups and their automorphism groups are easy to check, as it can be seen in the following Lemmas. The only exceptional case is $\operatorname{Aut}\left(A_{6}\right)$, which needs an ad-hoc argument.

Lemma 7.1. Let $A_{n}$ be the alternating group, with $n \geq 5$. Then $H:=$ $A_{n-1} \subset G$ is a cutting isotropy subgroup of $A_{n}$.

Proof. It suffices to consider the $(n-1)$-dimensional representation of $A_{n}$ : let $S$ be the set of $n$ elements on which $A_{n}$ acts. Then $\mathbb{R}[S]$ minus the trivial 
representation gives rise to the $(n-1)$-representation $V$ of $A_{n}$. The dimensions of the fixed subspaces $\operatorname{dim} V^{G}$ and $\operatorname{dim} V^{H}$ are 0 and 1 respectively. The subgroup $H$ is given by the elements in $A_{n}$ which fix one element in $S$, and it is easy to see that it is self-normalizing.

Lemma 7.2. If $G$ is a group such that $A_{n} \subset G \subset \operatorname{Aut}\left(A_{n}\right)$, with $n \geq 5$, then $G$ has a cutting isotropy subgroup.

Proof. If $n \neq 6$, then $\operatorname{Aut}\left(A_{n}\right)=\Sigma_{n}$ the $n$-symmetric group. Because of Lemma 7.1, we only need to prove it for $G:=\Sigma_{n}$, and the proof is identical to the alternating case. For $n=6$, let us consider $A_{6}$ acting on the 6 elements $s_{1}, \cdots, s_{6}$, and let $C_{5}$ be the cyclic group generated in $A_{6}$ by the 5cyclic permutation $\sigma$ of $s_{1}, \cdots, s_{5}$. It is a 5-Sylow group, and its normalizer is contained in $A_{5} \subset A_{6}$ (i.e., its normalizer fixes $s_{6}$ ). Therefore $N:=A_{6}$ is a normal subgroup of $G$ such such that the normalizer of its 5-Sylow is a cutting subgroup. By Lemma 5.3 we have the conclusion.

7.2. Groups of Lie type. In this section we just quote some well-known results in representation theory of simple groups of Lie type.

Lemma 7.3. Let $G$ be a simple group of Lie type. Then there exists a $p$ Sylow subgroup $P$ of $G$ such that its normalizer $N_{G}(P)$ is a cutting subgroup of $G$.

Proof. Let $V:=\mathrm{St}_{G}$ denote the real Steinberg representation of $G$ and $B$ the Borel subgroup of $G$. Then $\operatorname{dim} V^{G}=0$ because $\mathrm{St}_{G}$ is nontrivial and irreducible (cf. [Ca85], Corollary 6.2.4, page 190); moreover, $B$ is the normalizer in $G$ of a $p$-Sylow subgroup $U$ of $G$ (cf. [Ca85], Section 2.5, [St57], Section 4), and $\operatorname{dim} V^{B}=1$ because of Proposition 6.2.3, p. 190, of $[\mathbf{C a 8 5}]$. All the details on the Steinberg representation may be found in [St57, Cu66, CR87, Ca85].

Lemma 7.4. Let $G$ be a group such that $L \subset G \subset \operatorname{Aut}(L)$ where $L$ is a simple group of Lie type. Then $G$ has a cutting isotropy subgroup.

Proof. It follows from Lemma 7.3, applied to L, and then Lemma 5.3.

7.3. Sporadic groups. For the simple sporadic groups and their automorphism groups, it is enough to check the character tables, as given in [CC85]. The best and most easy way to do it, is to use GAP [Sc95], and its Character Table Libraries for simple groups. We refer to GAP Version 3 (Release 4.4). ${ }^{1}$

${ }^{1}$ Detailed information on GAP can be found in the following URL's:

http://www.math.rwth-aachen.de/ GAP/WWW (Germany),

http://www-gap.dcs.st-and.ac.uk/ gap (UK),

http://www.ccs.neu.edu/mirrors/GAP (USA) and

http://wwwmaths.anu.edu.au/research.groups/algebra/GAP/www (Australia). 
Lemma 7.5. If $G$ is an almost simple sporadic group, then it has a cutting isotropy subgroup.

Proof. We now give the GAP code of a function that can help in make computations on characters of groups, to detect cutting subgroups.

IsCutting: $=$ function $(\mathrm{G}, \mathrm{H}, \mathrm{fm})$

local tg, th, realchars, res, cut, trivialch;

$\operatorname{tg}:=$ CharTable $(G)$;

th: $=$ CharTable $(\mathrm{H})$;

trivialch:=List (th.classes, c $\rightarrow 1$ );

realchars: $=$ Filtered $($ tg. irreducibles, chi $\rightarrow$

Indicator (tg, $[\mathrm{chi}], 2)=[1])$;

if $\mathrm{fm}=[]$ then $\mathrm{fm}:=$ GetFusionMap (th,tg); fi;

res : $=$ Restricted (realchars, fm);

cut: $=$ Filtered (res, chi $\rightarrow$

ScalarProduct (th, trivialch, chi) $=1$ );

return Length(cut) $>1$;

end;

The variables $\mathrm{G}$ and $\mathrm{H}$ must be the names of the group $G$ and of the subgroup $H \subset G$, as given e.g., in Table 1. The variable $\mathrm{fm}$ is the fusion map from $H$ to $G$, given as a list (cf. [Sc95]). If $\mathbf{f m}$ is the empty list, then its value is the fusion map as recovered from the Character Table Libraries, by the function GetFusionMap (th,tg) where th is the source table, and tg is the destination table. The function IsCutting $(\mathrm{G}, \mathrm{H}, \mathrm{fm})$ returns true if $H$ is a cutting subgroup of $G$, and false otherwise. For example, to know whether M11M1 is a cutting subgroup of M11, we need to digit

gap>IsCutting ("M11" , "M11M1", [] );

true

gap>

The meaning of Table 1 is the following: For each sporadic group $S$ in the list, we have two cases: $\operatorname{Out}(S)=1$ or $\operatorname{Out}(S)=2$. In the first case, we only need to check for isotropy cutting subgroups of the group itself, and it turns out that among maximal subgroups there is always a cutting subgroup (which is therefore an isotropy subgroup). We give an example of such a maximal subgroup in the third column of the Table. On the other hand, if $\operatorname{Out}(S)=2$, this argument does not work in general, so we must use Lemma 5.3 and the tables of Sylow normalizers as given in [Os86], readable in GAP with the names listed in the second column. Those subgroups are the nontrivial Sylow normalizers in the simple group $S$, which already have a fusion map stored in the Character Table Libraries, except for the case of the Suzuki group Suz: in this case the (unique) fusion map must be explicitly recovered by the built-in function SubgroupFusions as follows: 
gap>fm:=SubgroupFusions (CharTable ("SuzN2" , "Suz")) [1] ; ; gap>IsCutting ("Suz" , "SuzN2" , fm);

true

gap>

\begin{tabular}{|c|c|c|c|c|}
\hline \multicolumn{2}{|c|}{ (GAP) Name } & Sylow Normalizer & Maximal subgroup & Out \\
\hline M11 & Mathieu & & $\mathrm{A} 6.2 \_3=\mathrm{M} 11 \mathrm{M} 1$ & 1 \\
\hline M12 & Mathieu & M12N2 & & 2 \\
\hline M22 & Mathieu & M22N2 & & 2 \\
\hline M23 & Mathieu & & $\mathrm{M} 22=\mathrm{M} 23 \mathrm{M} 1$ & 1 \\
\hline M24 & Mathieu & & $\mathrm{M} 23=\mathrm{M} 24 \mathrm{M} 1$ & 1 \\
\hline $\mathrm{J} 1$ & Janko & & $\mathrm{L} 2(11)=\mathrm{J} 1 \mathrm{M} 1$ & 1 \\
\hline J2 & Janko & J2N5 & & 2 \\
\hline J3 & Janko & J3N3 & & 2 \\
\hline J4 & Janko & & $\mathrm{mx} 1 \mathrm{j} 4=\mathrm{J} 4 \mathrm{M} 1$ & 1 \\
\hline Co1 & Conway & & $\mathrm{Co} 2=\mathrm{Co} 1 \mathrm{M} 1$ & 1 \\
\hline Co2 & Conway & & $\mathrm{U} 6(2) .2=$ Co2M1 & 1 \\
\hline Co3 & Conway & & McL.2 = Co3M1 & 1 \\
\hline Fi22 & Fischer & Fi22N5 & & 2 \\
\hline Fi23 & Fischer & & 2.Fi22 = Fi23M1 & 1 \\
\hline F3+ & Fischer & & Fi23 (*) & 2 \\
\hline HS & Higman-Sims & HSN2 & & 2 \\
\hline McL & McLaughlin & McLN5 & & 2 \\
\hline $\mathrm{He}$ & Held & HeN7 & & 2 \\
\hline $\mathrm{Ru}$ & Rudvalis & & $2 \mathrm{~F} 4(2)^{\prime} \cdot 2=$ RuM1 & 1 \\
\hline Suz & Suzuki & SuzN2 & & 2 \\
\hline $\mathrm{ON}$ & O’Nan & ONN3 & & 2 \\
\hline HN & Harada-Norton & & $2 . \mathrm{HS} .2=$ HNM2 $(*)$ & 2 \\
\hline Ly & Lyons & & $\operatorname{GL}(5)=$ LyM1 & 1 \\
\hline Th & Thompson & & $3 \mathrm{D} 4(2) \cdot 3=\mathrm{ThM} 1$ & 1 \\
\hline B & Baby Monster & & Fi23 & 1 \\
\hline M & Monster & & $2 . B$ & 1 \\
\hline
\end{tabular}

Table 1. Examples of cutting subgroups of sporadic simple groups.

So, only F3+ and HN, (marked with $(*)$ ) are left, because there are no such tables for their Sylow normalizers: In this case we must use Lemma 5.4, and the fact that Fi23 and HNM2 are maximal subgroups of F3+ and HN which are intersections of maximal subgroups in Aut(F3+) and Aut(HN) (cf. [NW86], [LW91]).

Therefore the conclusion of the Lemma follows by applying the function IsCutting to the list of groups and subgroups in Table 1. Let use note that these are only examples of cutting subgroups: As it is readily seen, most of 
the maximal subgroups and Sylow normalizers are cutting subgroups. The groups in the list are the ones which are more or less ready to use in the Character Table Libraries.

\section{Non 2-split groups.}

We are now able to prove the last proposition, which is equivalent to (ii) $\Longrightarrow(\mathrm{i})$.

Proposition 8.1. If $G$ is a finite group which is not 2-split, then it has a cutting isotropy subgroup.

Proof. Let us assume on the contrary that there is at least one group not 2 -split and without cutting isotropy subgroups. Let $G$ be the smallest group of this type (with respect to the order). Because of the Lemmas in Section 7, $G$ cannot be simple, hence there is a nontrivial minimal normal subgroup $1 \neq N \triangleleft G$; because of minimality and Lemma $5.1, G / N$ must be 2-split, hence solvable. Therefore $N$ is a direct product of $n$ copies of a finite simple group $S$, say $N \cong S^{n}=S \times \times \cdots \times S$. Moreover, $C_{G}(N)=1$ : In fact, if $C_{G}(N) \neq 1$ by the same minimality argument we obtain that $G / C_{G}(N)$ is 2-split and hence solvable, which contradicts the fact that $S$ is simple non-abelian. Therefore $G$ is contained in $\operatorname{Aut}(N) \cong \operatorname{Aut}(S)^{n} \rtimes \Sigma_{n}$, where $\Sigma_{n}$ denotes the $n$-th symmetric group.

$$
N=S^{n} \triangleleft G \subset \operatorname{Aut}(S)^{n} \rtimes \Sigma_{n} .
$$

Because $N$ is minimal in $G, G \cap \operatorname{Aut}(S)^{n}$ must be of the form $G \cap \operatorname{Aut}(S)^{n} \cong$ $G_{0}^{n}$, for a subgroup $G_{0} \subset \operatorname{Aut}(S)$ such that $G_{0} \supset S$. In other words, $G_{0}$ is almost simple, and hence by applying the results of Section 7 it has a cutting isotropy subgroup $H_{0}$. By taking the $n$-th power of the tensor product of the representation of $G_{0}$, we can see that $H_{0}^{n}$ is a cutting isotropy subgroup of $G_{0}^{n}$. Furthermore, $G_{0}^{n}$ is a normal subgroup of $G$, and there exists a subgroup $H$ of $G$ such that $H \cap G_{0}^{n}=H_{0}^{n}$ and $H G_{0}^{n}=G$. Therefore we can apply Lemma 5.2, to prove that $H$ is a cutting isotropy subgroup of $G$. But this is a contradiction, because we assumed $G$ to have no cutting isotropy subgroups, hence the thesis.

\section{References}

[Br72] G.E. Bredon, Introduction to compact transformation groups, Academic Press, New York London, 1972.

[Ca85] R.W. Carter, Finite groups of Lie type, John Wiley \& Sons. Chichester-New York, 1985.

[CC85] J.H. Conway, R.T. Curtis, S.P. Norton, R.A. Parker and R.A. Wilson, Atlas of finite groups, Oxford University Press, 1985.

[Cu66] C.W. Curtis, The Steinberg character of a finite group with a BN-pair, J. Algebra, 4 (1966), 433-441. 
[CR87] C.W. Curtis and I. Reiner, Methods of representation theory, II, John Wiley \& Sons, 1987.

[Do72] A. Dold, Lectures on Algebraic Topology, Springer Verlag, Berlin, Heidelberg, New York, 1972.

[FW88] E. Fadell and P. Wong, On deforming G-maps to be fixed point free, Pac. J. of Math., 132(2) (1988), 277-281.

[Ja85] N. Jacobson, Basic algebra I, 2nd ed., W.H. Freeman and Company, New York, 1985.

[LW91] S.A. Linton and R.A. Wilson, The maximal subgroups of the Fischer groups Fi $i_{24}$ and $F i_{24}^{\prime}$, Proc. Lond. Math. Soc., III Ser. 63, No. 1, (1991), 113-164.

[NW86] S.P. Norton and R.A. Wilson, Maximal subgroups of the Harada-Norton group, J. Algebra, 103 (1986), 362-376.

[Os86] T. Ostermann, Charaktertafeln von Sylownormalisatoren sporadischer einfacher Gruppen, Vorlesungen aus dem Fachbereich Mathematik 14, Universität Essen, 1986.

[Ro82] D.J.S. Robinson, A Course in the Theory of Groups, G.T.M. 80, Springer-Verlag, New York Heidelberg Berlin, 1982.

[Sc95] M. Schönert et al., GAP - Groups, Algorithms and Programming, Lehrstuhl D für Mathematik, Rheinisch Westfälische Technische Hochschule, Aachen, Germany, fifth edition, 1995.

[Se77] J.P. Serre, Linear Representations of Finite Groups, Graduate Texts in Mathematics, 42, Springer-Verlag, New York, Heidelberg, Berlin, 1977.

[St57] R. Steinberg, Prime power representations of finite groups, II, Canadian J. of Math., 9 (1957), 347-351.

[Su82] M. Suzuki, Group Theory, I, Grundlehren der mathematischen Wissenschaften 247, Berlin, Heidelberg, New York, Springer Verlag, 1982.

[tD87] T. tom Dieck, Transformation groups, Walter de Gruyter, Berlin, New York, 1987.

[Wi84] D. Wilczyński, Fixed point free equivariant homotopy classes, Fundamenta Mathematicae, 123 (1984), 47-60.

[Wo91] P. Wong, Equivariant Nielsen fixed point theory for G-maps, Pac. J. of Math., 150(1) (1991), 179-200.

Received November 19, 1998.

Dipartimento di Matematica

del Politecnico di Milano

Piazza Leonardo da Vinci, 32

20133 Milano

ITALY

E-mail address: ferrario@mate.polimi.it 